\title{
EDITORIAL
}

\section{Controversial topics in tuberculosis}

\author{
J.A. Caminero*, A. Torres ${ }^{\#}$
}

Tuberculosis (TB) is certainly the disease that has provoked the most damage to mankind throughout history. It has caused death and disease for perhaps $>20,000$ yrs [1] and, as a rule, has affected the poorest strata of society. Such a long time of common life with men has endowed Mycobacterium tuberculosis, the causal agent, with the best adaptation among all known human pathogens. Therefore, it has remained in a quiescent state within a large number of individuals, generating neither symptoms nor disease, but surviving and awaiting more suitable conditions to attack [2]. Today, it is estimated that one-third of the global population, around 2,000 million people [3], lives with this microorganism, known as Koch's bacillus, and represents the largest reservoir of healthy, infected carriers for any given infectious disease. It is disconcerting that, at the onset of a new millennium and after so numerous and significant developments of the last century, one has to admit that TB remains the major infectious disease by the number of healthy infected persons in the world, as well as by the 8 million people with the disease, and the 2 million deaths it still causes each year [3]. A number of reasons could explain this worrying situation, but ahead of all of these is the enormous inequality in the global distribution of wealth, which constantly increases the fraction of people living in extreme poverty, the most suitable condition for TB dissemination [2]. Moreover, the burden of AIDS has further degraded the situation in areas where TB had not been effectively controlled [2-4]. In addition, there is great disparity in the global distribution of TB. Some wealthy countries anticipate a probable elimination of TB in the next few decades, while poor nations witness a worsening of their problem every year, in spite of the resources devoted to TB control $[2,3]$. Europe is a good example of this disparity, since some western countries report low or medium incidence and a favourable evolution in the last decade, whereas some eastern countries show much higher disease rates and a borneout increase during the last 10 yrs [3, 4].

TB is, perhaps, the most written about disease in the history of medicine [2]. It is significant that TB was determinant at the origin not only of pulmonary medicine and thoracic surgery, but also of other medical specialities. Nevertheless, looking at this knowledge from a historic point of view, one realises that empiricism has prevailed until relatively recent times [2]. Up until 1882 the cause of the disease remained unknown, and some defended the theory of its inherited origin. However, research on TB during the twentieth century has yielded multiple and major developments, indeed at a slower pace during the last $10 \mathrm{yrs}$, than research on other diseases. The fact that TB is a disease of the poor has always played against its chances for research investment. Nevertheless, important knowledge has been acquired regarding its epidemiological behaviour, its virulence and pathogenesis, and also concerning

*Dept of Pulmonary Medicine, Hospital General de Gran Canaria "Dr. Negrín", Las Palmas de Gran Canaria, "Dept of Pulmonary Medicine, Hospital Clinic, Barcelona, Spain.

Correspondence: J.A. Caminero, Neumología, Hospital General de Gran Canaria, Barranco de la Ballena s/n, Las Palmas 35010, Spain. Fax: 34 928450085. E-mail: jcamlun@gobiernodecanarias.org potential diagnostic, therapeutic and control procedures. In spite of this significant progress, a critical look will disclose the following major deficiencies: 1) the basis of the current pathogenic rationale is $>40$ yrs old; 2 ) the diagnosis still depends on sputum microscopy and culture, which has been in use for $>120 \mathrm{yrs}$; and 3) rifampin, the last drug included in initial treatment schemes, was developed back in 1963. Nevertheless, the message should be optimistic, since we face a disease that is simple to diagnose in advanced forms (sputum microscopy) and curable in nearly all cases with treatment regimens that, although inconveniently long, cost no more than $€ 10$.

A significant amount of TB research, carried out during the last decades, has either yielded knowledge only applicable to wealthy countries [2], which endure the smallest share of the burden (the industrialised countries contribute altogether to only $6 \%$ of the global cases of tuberculosis [3]), or has been insufficiently verified. Moreover, some of this new research has challenged previously well-accepted knowledge about TB. Some of these controversial issues are currently of great interest, fully justifying the publication of a series in which the most important and debatable topics will be in-depth reviewed and analysed by suitable experts. The current series analyses the following nine interesting topics: 1) on the nature of $M$. tuberculosis-latent bacilli [5]; 2) annual risk of infection with $M$. tuberculosis; 3) nonconventional and new methods in the diagnosis of TB: feasibility and applicability in the field; 4) drug resistance in TB; 5) drugs susceptibility test in TB: methods, positive predictive value and interpretation of the results; 6) should TB treatment and control be addressed differently in HIV-infected and uninfected individuals?; 7) management of multi-drug-resistant TB and patients in re-treatment; 8) cost-effectiveness of TB control strategies among immigrants and refugees; and 9) the dream of a new vaccine against TB: improving or replacing bacille Calmette-Guérin?

We hope that this series will elucidate doubts on each of the treated subjects and will raise awareness to encourage the necessary research in the tuberculosis field, both a simple and complex area of knowledge.

\section{References}

1. Daniel TM. The origins and precolonial epidemiology of tuberculosis in the Americas: can we figure them out? Int J Tuberc Lung Dis 2000; 4: 395-400.

2. Caminero JA. Tuberculosis guide for specialist physicians. Paris, International Union against Tuberculosis and Lung Disease, 2004.

3. World Health Organization. Global tuberculosis control. WHO report 2003 (WHO/CDS/TB/2003.316). Geneva, World Health Organization, 2003.

4. De Colombani P, Banatvala N, Zaleskis R, Maher D, WHO regional office for Europe. European framework to decrease the burden of TB/HIV. Eur Respir J 2004; 24: 493-501.

5. Cardona P-J, Ruiz-Manzano J. On the nature of Mycobacterium tuberculosis-latent bacilli. Eur Respir J 2004; 24: 1044-1051. 